\section{Duodenal ulcer caused by a surgical clip after laparoscopic cholecystectomy}

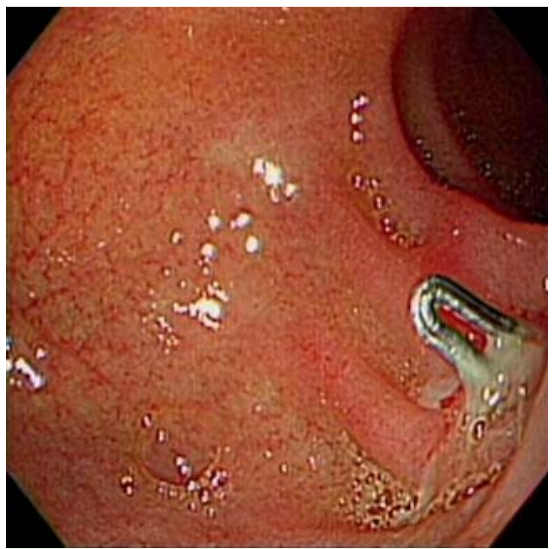

Fig. 1 Esophagogastroduodenoscopy (EGD) revealed an ulcer with a wire-like object protruding from it in the duodenal bulb.
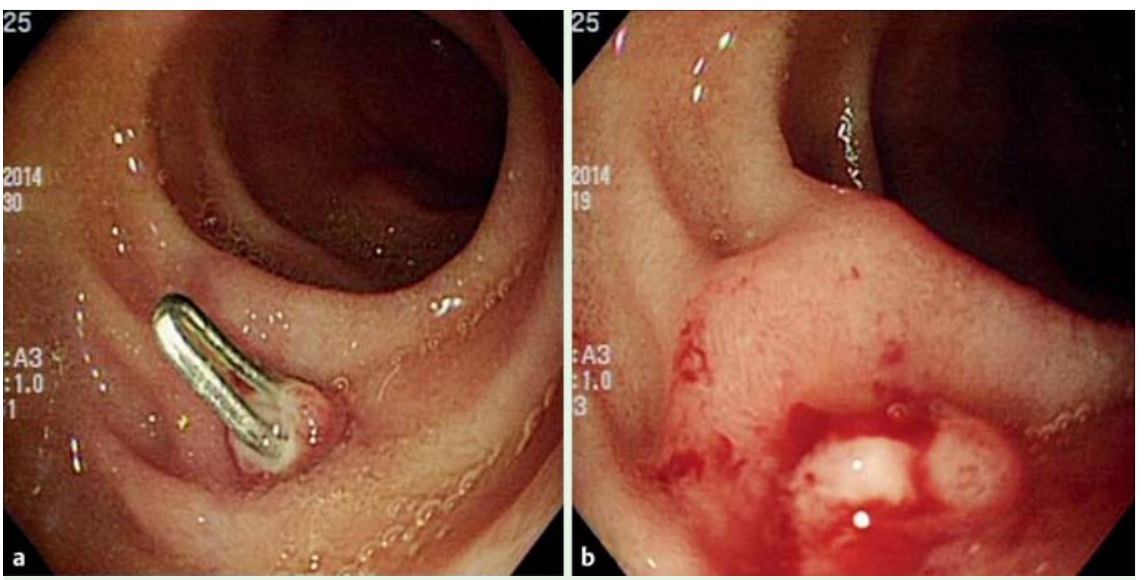

Fig. 3 Views during repeat esophagogastroduodenoscopy (EGD) performed 1 month later showing: a improvement in the appearance of the ulcer, but further protrusion of the clip; b mild bleeding after the clip had been retrieved using biopsy forceps.

A 56-year-old woman presented with a 1-month history of right upper quadrant abdominal pain after a laparoscopic cholecystectomy for gallstones. Her medical history included endoscopic retrograde cholangiopancreatography (ERCP) performed 4 months prior to the laparoscopic cholecystectomy for the treatment of common bile duct (CBD) stones. During this procedure duodenal ulcers had also been found at the duodenal bulb and she had therefore taken proton pump inhibitors (PPIs) for 3 months.

Physical examination revealed tenderness in the right upper quadrant of her abdomen. Abdominal ultrasonography showed that the bile ducts were not dilated. Esophagogastroduodenoscopy (EGD) revealed an ulcer with a wire-like object in

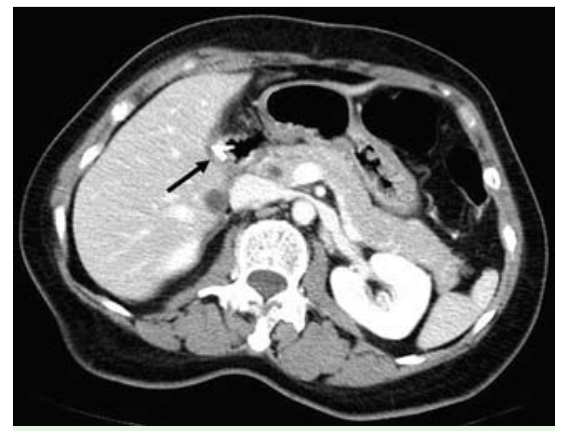

Fig. 2 A contrast-enhanced abdominal computed tomography (CT) scan showed part of a surgical clip (arrow) in the lumen of the postbulbar zone of the duodenum, indicating bowel penetration.

inal computed tomography (CT) scan showed that part of a surgical clip was located in the lumen of the postbulbar zone of the duodenum ( $\bullet$ Fig.2). A duodenal ulcer resulting from a surgical clip placed during the laparoscopic cholecystectomy was diagnosed.

The patient's abdominal pain subsided gradually after further PPI use. An EGD performed 1 month later showed that the ulcer had improved, but the clip was protruding further ( $\bullet$ Fig.3a). The clip was retrieved using biopsy forceps, which led to mild bleeding ( $\bullet$ Fig.3 b), and a hemoclip was applied to close the ulcer. The patient continued using PPIs and subsequently experienced an uneventful course.
Surgical clip-related complications after laparoscopic cholecystectomy can include clip migration into the CBD, bile leak, and clip embolism [1]. A surgical clip-related duodenal ulcer is a rare complication after laparoscopic cholecystectomy and may be caused by two proposed mechanisms [2]. First, as the clip lies adjacent to the duodenum and may directly erode the duodenal wall, it emerges in the base of the ulcer. Second, a pre-existing ulcer can transiently perforate and capture the clip during the healing process.

The management of a surgical clip that is present in a duodenal ulcer remains controversial. Although spontaneous detachment of such clips has been reported [3], endoscopic clip removal is recommended [1].

\section{Endoscopy_UCTN_Code_CPL_1AN_2AG}

\section{Competing interests: None}

\section{Ming-Che Hsin ${ }^{1}$, I-Chang Lin², Chi-Ming Tai ${ }^{3}$}

${ }^{1}$ Department of Surgery, E-Da Hospital, I-Shou University, Kaohsiung, Taiwan

2 Department of Radiology, E-Da Hospital, I-Shou University, Kaohsiung, Taiwan

${ }^{3}$ Department of Internal Medicine,

E-Da Hospital, I-Shou University,

Kaohsiung, Taiwan

\section{References}

1 Wasserberg N, Gal E, Fuko Z et al. Surgical clip found in duodenal ulcer after laparoscopic cholecystectomy. Surg Laparosc Endosc Percutan Tech 2003; 13: 387-388

2 Heatley MK, Nagarajan DV. The ulcer and the clip: which came first? Gut 2002; 50: 129

3 Yu SC, Yuan RH, Ho MC et al. Duodenal Endoclip migration after laparoscopic cholecystectomy: report of a case. Endoscopy 1997; 29: 339

\section{Bibliography}

DOI http://dx.doi.org/

10.1055/s-0034-1391869

Endoscopy 2015; 47: E264

(c) Georg Thieme Verlag KG

Stuttgart · New York

ISSN 0013-726X

\section{Corresponding author}

\section{Chi-Ming Tai, MD}

Department of Internal Medicine E-Da Hospital

1, E-Da Rd, Jiau-Shu Village

Yan-Chau District

Kaohsiung 824

Taiwan ROC

chimingtai@gmail.com 